\title{
Cyclooxygenase-2 expression in actinic keratosis
}

\author{
Kinga Adamska ${ }^{1}$, Mariola Pawlaczyk², Monika Bowszyc-Dmochowska ${ }^{3}$, Justyna Gornowicz-Piotrowska , \\ Małgorzata Janicka-Jedyńska ${ }^{5}$, Tomasz Fedorowicz ${ }^{6}$, Ryszard Żaba ${ }^{1}$
}

\begin{abstract}
${ }^{1}$ Department of Dermatology and Venereology, Poznan University of Medical Sciences, Poznan, Poland ${ }^{2}$ Department of Geriatric Medicine and Gerontology, Poznan University of Medical Sciences, Poznan, Poland ${ }^{3}$ Cutaneous Histopathology and Immunopathology Section, Department of Dermatology, Poznan University of Medical Sciences, Poznan, Poland

${ }^{4}$ Autoimmune Blistering Dermatoses Section, Department of Dermatology, Poznan University of Medical Sciences, Poznan, Poland ${ }^{5}$ Department of Clinical Pathology, Poznan University of Medical Sciences, Poznan, Poland

${ }^{6}$ Diagnostic Center of Skin Nevi, Poznan, Poland
\end{abstract}

Adv Dermatol Allergol 2018; XXXV (6): 626-630

DOI: https://doi.org/10.5114/ada.2018.72663

\begin{abstract}
Introduction: Actinic keratosis (AK) is a common age-associated dermatosis typical for skin with photo-ageing features. Actinic keratosis lesions transform into invasive squamous cell carcinoma, if left untreated, but inductive factors remain unknown. A role of prostaglandins in the neoplastic process has been postulated.

Aim: To evaluate the possible correlation between cyclooxygenase-2 (COX-2) antigen expression in the immunohistochemical reaction and the stage of AK using the keratinocytic intraepidermal neoplasia (KIN) classification, age, sex and skin phenotype.

Material and methods: Skin samples of AK were examined histopathologically using the KIN classification. Immunohistochemical analysis of COX-2 expression was conducted using a commercially available kit and Image Processing and Analysis in Java. The intensity was presented as the percentage of cells with a positive reaction.

Results: Out of the 94 subjects with AK (aged 51-93 years; mean: $76.51 \pm 9.64$ ), 58 were female and 36 male. Phenotype 2 was found in 50 and phenotype 3 in 44 patients. Mean values of positive immunohistochemical reaction for COX-2 were $2.16 \pm 6.56 \%$ for women and $1.96 \pm 6.59 \%$ for men $(2.47 \pm 6.61 \%)$, with no statistically significant difference. Mean values of anti-COX-2 antibody reaction were $2.30 \pm 6.82 \%$ in the KIN1 group and $2.48 \pm 7.01 \%$ in the KIN2 group, while no expression was found in all cases with KIN3. No statistically significant differences were found between the percentage of COX-2 positive cells in KIN1/KIN2.

Conclusions: No statistically significant correlations between the intensity of COX-2 reaction and AK stage were found. Cyclooxygenase-2 expression in AK is not affected by age, sex, or skin phenotype.
\end{abstract}

Key words: actinic keratosis, cyclooxygenase-2, histopathology.

\section{Introduction}

The aging process of human skin is associated not only with undesired esthetic changes but also the development of age-associated dermatoses, with actinic keratosis (AK), also known as solar keratosis, among the most common problems. Clinical presentation of AK consists in round or oval yellow-brown plaques of scaly or crusty erythematous skin, typically on the skin, with photo-aging features. Sites of predilection include the face, ears, bald scalp and dorsal parts of hands and forearms [1-3]. Actinic keratosis is an example of a pre-cancerous lesion, with a malignant potential to transform into invasive squamous cell carcinoma (SCC) [3]. Based on cytological and biomolecular testing, AK is considered to be a case of "in situ squamous carcinoma type actinic keratosis", which may develop into an invasive form [3].

The incidence of AK increases with age, proximity to the Equator, lighter skin and outdoor activity. The highest prevalence has been reported in Australia: 79\% and $68 \%$ of males and females aged $60-69$, respectively, but only $10 \%$ of men and $5 \%$ of women aged $20-29$ years, respectively, indicating that the disease is sex and agerelated $[4,5]$. Prolonged exposure to ultraviolet radiation (UV) - mainly UVB, which has the highest carcinogenic potential, as well as pale skin phenotype - typical for the Caucasian race, play the key roles in the etiopathogenesis of AK [6-9].

Address for correspondence: Kinga Adamska MD, PhD, Department of Dermatology and Venereology, Poznan University of Medical Sciences, 49 Przybyszewskiego St, 60-355 Poznan, Poland, phone: +48 507770 763, e-mail: kingabyczkowska@gmail.com Received: 10.11.2017, accepted: 29.11.2017. 
Theoretically, AK lesions may transform into invasive SCC, if left untreated, but the exact time span and inductive factors remain unknown. Also, approximately 25\% of AK lesions will spontaneously regress and the contributing factors also remain to be elucidated [10]. Further studies are necessary to identify the disease stage which marks the irreversibility of the skin changes. Out of numerous classifications for solar keratosis, the keratinocyte intraepidermal neoplasia (KIN) classification developed by Cockerell et al. has proven to be the most useful system, as it takes into consideration the histopathological features (Table 1), and corresponds to the cervical intraepithelial neoplasia (CIN) and vulvar intraepithelial neoplasia (VIN) classifications used in gynecology [11].

Prostaglandins play a significant role as modulators of the immune reaction in inflamed or cancerous tissues [12]. Cyclooxygenase-2 (COX-2) is one of the catalysts for the arachidonic acid to prostaglandin conversion process. The exact mechanism of COX-2's role in the neoplastic process had not been fully explained. The enzyme blocks cell apoptosis in the tumor and directly affects its expansion by inducing angiogenesis and weakening the function of T-lymphocytes and NK cells, which has been demonstrated by in vitro studies [12].

\section{Aim}

The aim of the study was to evaluate the possible correlation between COX-2 antigen expression in immunohistochemical (IHC) reaction and the stage of AK using the KIN classification system, age, sex and skin phenotype.

\section{Material and methods}

A total of 94 Caucasian patients with AK were included in the study. Skin samples of $\leq 5 \mathrm{~mm}$ in diameter were excised from all subjects for histopathological and immunohistochemical analysis. The samples were fixed in 10\% buffered-formalin solution and embedded in paraffin. Next, they were cut into $4 \mu \mathrm{m}$ sections and mounted on simple adhesive slides and stained with hematoxylin and eosin $(\mathrm{H}+\mathrm{E})$. The preparations were analyzed using an Olympus BX 40 optical microscope. The epidermal architectural and cytological features of AK were investigated including: epidermal thickness, hyper- and parakeratosis, hypercellularity, loss of stratification, abnormal keratinocyte maturation, presence and level of cellular atypia, namely enlarged, hyperchromatic, irregular nuclei and mitoses. Three AK classification stages - KIN1, KIN2, and KIN3 - were used in the histopathological evaluation [9].

Immunohistochemical analysis of COX-2 expression in paraffin samples was conducted using the commercially available EnVision Flex kit (Dako, Denmark). In order to facilitate antibody penetration, the samples underwent heat-based antigen retrieval using Target Retrieval Solution, High pH (Dako, Denmark) at $95-99^{\circ} \mathrm{C}$ in a water bath for 90 min. Next, a 3\% hydrogen peroxide (Peroxidase block) was applied to block the activity of endogenous peroxidase and incubated for $5 \mathrm{~min}$ in a wet chamber at room temperature. Monoclonal mouse anti-human protein COX-2 clone CX-294 antibody (Dako, Denmark) at $1: 100$ dilution was used. The stained preparations were evaluated using an Olympus BX 40 optical microscope with a magnification grade of 200x. Digital photos were taken with a CamediaC-2002 digital camera and quantitatively analyzed using ImageJ (Image Processing and Analysis in Java) (http://imagej.nih.gov/ij/). Regions of intensive reaction and characteristic histopathologic features, free of imaging artefacts, were selected for the numerical analysis of $\mathrm{IHC}$ reactions. ImageJ software was used to localize regions of interest (ROI) localized in the skin, segmentation using color thresholding was performed and the nuclei with positive reaction were selected. Stained nuclei are often interconnected, so segmentation using morphological watersheds was applied to calculate their number. Next, nuclei with a negative reaction were identified in the same image and using the same segmentation methods. IHC intensity was presented as the percentage of cells with a positive reaction.

\section{Statistical analysis}

Statistical analysis was performed using Statistica PL 10.0. The Shapiro-Wilk test was used to test for the normality of data. In the absence of normal distribution, the obtained results were presented using mean, standard deviation, median and range of the parameters. The Mann-Whitney test for independent variables was used to compare two groups of qualitative variables. The

Table 1. Results of histopathology with KIN classification

\begin{tabular}{|c|c|c|c|}
\hline KIN stage & $\begin{array}{l}\text { Total } \\
N(\%)\end{array}$ & $\begin{array}{l}\text { Women } \\
n(\%)\end{array}$ & $\begin{array}{l}\text { Men } \\
n(\%)\end{array}$ \\
\hline $\begin{array}{l}\text { 1: keratinocyte atypia confined to bottom third of the epithelium with basal and suprabasal } \\
\text { cells showing some nuclear enlargement and hyperchromasia }\end{array}$ & $32(34)$ & $21(36)$ & $11(31)$ \\
\hline 2: atypia involving the lower two-thirds of the epidermis & $52(56)$ & $31(53)$ & $22(61)$ \\
\hline $\begin{array}{l}\text { 3: carcinoma in situ with full-thickness atypia involving the epidermis and adnexal } \\
\text { structures }\end{array}$ & $10(11)$ & $7(12)$ & $3(8)$ \\
\hline
\end{tabular}




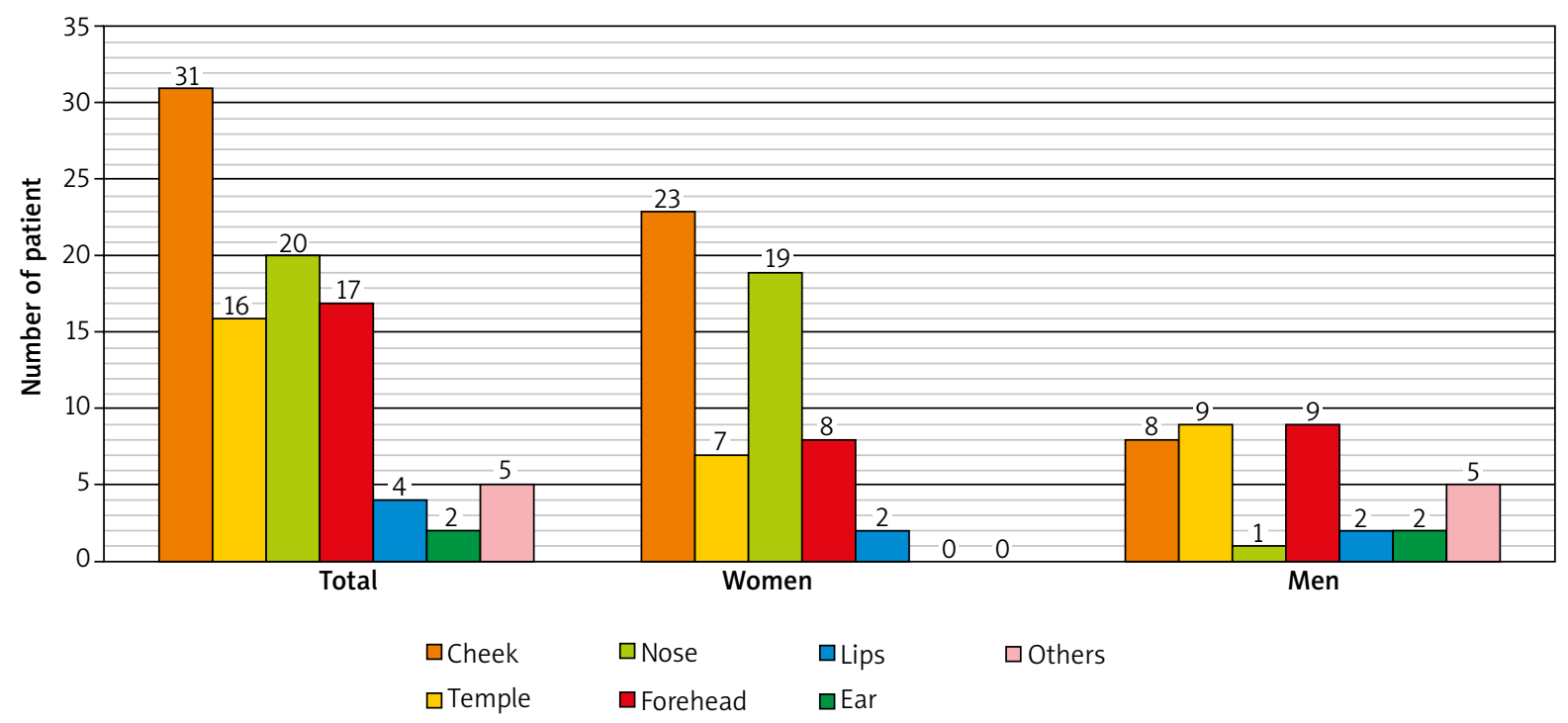

Figure 1. Number of patients with AK in different locations

inter-variable differences, depending on the KIN classification, were tested first with the Kruskal-Wallis and next with the multiple comparison test. Spearman's rank correlation was used to test for dependencies. A $p$-value of $<0.05$ was considered as statistically significant. Local Ethics Committee approved of the study (no. 153/14). Informed consent was obtained from all study participants.

\section{Results}

Out of the 94 subjects with AK (aged: 51-93 years; mean: $76.51 \pm 9.64)$, 58 were female (aged: $51-93$ years; mean: $76.31 \pm 10.10$ ), and 36 were male (aged: 51-93 years; mean: $76.83 \pm 8.98)$. Phenotypes 2 and 3 were found in 50 (54\%) and 44 (46\%) patients, respectively. Location of AK lesions is presented in Figure 1. The number of women with AK located on the nose was statistically significantly higher as compared to men ( $p=0.0008)$. No statistically significant sex-related differences were found for other locations. Table 1 presents the AK classification into three KIN stages, in accordance with histopathology results. Mean values of positive $\mathrm{IHC}$ reaction for COX-2 were $2.16 \pm 6.56 \%$ for women and $1.96 \pm 6.59 \%$ for men $(2.47 \pm 6.61 \%)$. No statistically significant difference was found ( $p=0.7747$ ). Figure 2 presents COX-2 antigen expression in AK preparation. Mean values of anti-COX-2 antibody reaction were $2.30 \pm 6.82 \%$ and $2.48 \pm 7.01 \%$ in the KIN1 and KIN2 groups, respectively, while no COX-2 expression was found in any of the 10 cases in the KIN3 group. No statistically significant difference was found between the percentage of positive cells for the COX-2 antibody in stage 1 and $2 \mathrm{KIN}(p=0.4848)$ (Figure 3). Mean values of positive reactions with the anti-COX-2 antibody in the phenotype 2 and 3 groups were 1.41 $\pm 0.61 \%$ and $3.02 \pm 8.25 \%$, respectively. No statistically significant difference was found ( $p=0.7020$ ) (Figure 4). No difference in COX-2 expression was found with regard to sex $(p=0.7747)$. Spearman's correlation test revealed no statistically significant correlations between the variables $(p>0.05)$, which indicates that age, sex, skin phenotype and KIN stage did not affect the percentage of positive reactions with the anti-COX-2 antibody.

\section{Discussion}

The diagnostic process for AK is predominantly based on the clinical evaluation and presents little, if any, challenge for an experienced clinician. However, differentiation between AK and invasive SCC on the basis of dermatology may pose a difficulty. Histopathological evaluation following skin biopsy remains the gold standard.

The role of prostaglandins in the neoplastic process, which has been postulated by numerous authors, is based on the efficacy of prostaglandin inhibitors in the process of inhibiting metastatic or tumor growth, which has been demonstrated in in vitro studies. Inhibition of the COX-2 activity is the basic mechanism of anti-inflammatory action of non-steroidal-anti-inflammatory drugs (NSAIDs), initially used only in the treatment for rheumatoid arthritis [13]. Non-steroidal-anti-inflammatory drugs have been demonstrated to have a chemopreventive effect on colorectal cancer [14], owing to their direct inhibitory effect on cyclooxygenase induction, and attempts have been made to use these drugs in the therapy of adenocarcinoma and colorectal cancer $[13,15]$. Also, they have been successfully applied in oncological dermatology as studies in vitro and in vivo animal models have 
shown high effectiveness of cyclooxygenase inhibitors in skin carcinogenesis [16].

Anuja et al. emphasized the key role of chronic inflammation in the induction of neoplastic growth and the fact that it negatively impacts genomic stability [17], while Nissinen et al. demonstrated that disturbances in the tumor microenvironment of SCC are induced by the inflammation and consist in the influx of inflammatory cells which promote protease synthesis, dysregulation of cytokine and chemokine secretion, which in turn leads to changes in the basement membrane and progression of early cancerous lesions to invasive forms [18].

Data on COX-2 expression in skin cancers remain inconclusive. Kagoura et al., in their study on selected skin cancers - 16 cases of basal cell carcinoma (BCC), 15 SCC, 10 Bowen's disease (BD) and 15 metastases to the skin - demonstrated lack of expression in 12 out of 16 BCC samples and moderate expression in the remaining 4 samples. As for BD, moderate expression was observed in 10 out of 13 samples. Positive $\mathrm{IHC}$ reaction, although of varying intensity, was demonstrated in 11 out of 15 SCC samples. Only 2 (well-differentiated pulmonary adenocarcinoma) out of 15 metastatic tumors showed cOX-2 expression [19].

The presence of COX-2 in human epidermis and its increased synthesis in inflammatory or cancerous states have both been demonstrated in in vitro and in vivo studies. Buckman observed a 6 -fold higher increase of that enzyme in keratinocytes after UVB treatment in laboratory conditions [20]. Also, they found a statistically significant difference in COX-2 staining in samples obtained from squamous-cell skin carcinoma sites as compared to healthy skin samples, not exposed to ultraviolet radiation. Their results might suggest a link between inflammatory processes and induction of the malignant transformation in skin after exposure to UV, so increased COX-2 expression in histopathologically more advanced stages of AK could be expected, which is not consistent with our findings. Variable COX-2 expression was also observed in other skin diseases and conditions. Kuźbicki et al. investigated COX-2 expression in healthy skin and 20 benign epithelial changes, including seborrheic keratosis, 11 pre-cancerous lesions, 35 cancerous lesions (21 BCC and 14 SCC), and 6 skin tags. These authors demonstrated that COX-2 expression in the benign changes was similar to healthy skin and was highest in the granular layer and lower in the spinous layer, with the gradient increasing toward the skin surface. Higher COX-2 expression was observed in the pre-cancerous as compared to benign lesions. In contrast to the healthy skin samples and benign lesions, expression in the keratinocytes of the basal layer of the epidermis was detected. Interestingly, low COX-2 expression was found in BCC samples, and it was lower compared to benign changes, unlike in SCC samples, where COX-2 expression was high, reaching the pre-cancerous lesion values. Of note, no differ-

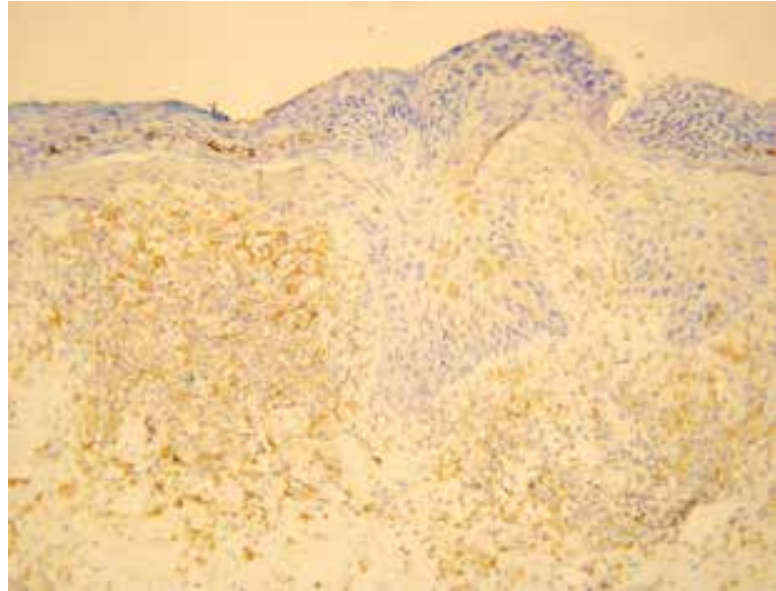

Figure 2. Positive immunohistochemical reaction with anti-COX-2 antibody in AK lesion located on the cheek of a 76-year-old woman (original magnification 200x)

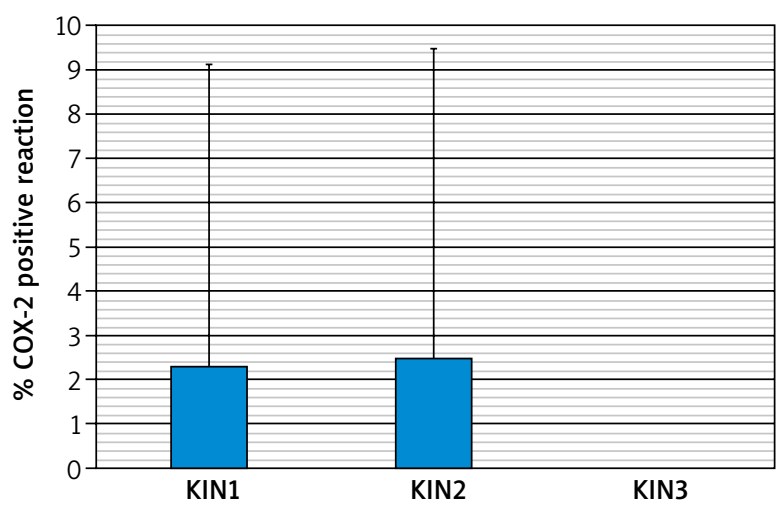

Figure 3. Percentage of positive reactions with anti-COX-2 antibody in three KIN stages

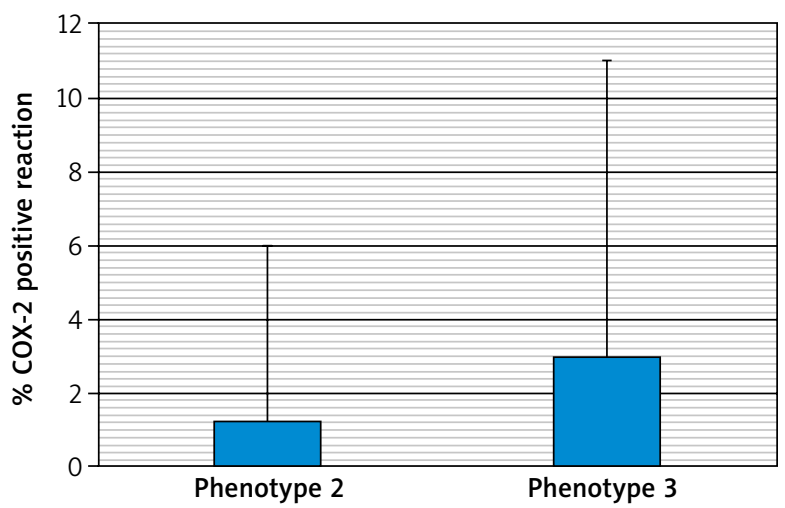

Figure 4. Positive IHC reaction for COX-2 expressed as percentages, depending on skin phenotype

ences in COX-2 expression between various stages of SCC were observed. No COX-2 expression was detected in fibroid samples [21]. A similar study was conducted by Amirnia et al., who analyzed 17 SCC, 32 BCC, 9 BD 
and 4 AK cases and found COX-2 expression in 16 out of 17 SCC, 28 out of 32 BCC, 8 out of 9 BD, and all 4 AK skin samples. They did not analyze the localization of COX-2 expression in the epidermal layers of the investigated biopsy specimens [22]. Our findings indicated that distinct COX-2 expression in KIN1 and KIN2 disappears in KIN3, the most advanced stage, which might suggest subsiding inflammation.

\section{Conclusions}

No statistically significant correlations between the intensity of $\mathrm{IHC}$ reaction and disease stage (KIN classification) were found. Antigen COX-2 expression in AK skin samples is not affected by age, sex, or skin phenotype of the patients.

\section{Conflict of interest}

The authors declare no conflict of interest.

\section{References}

1. Goldberg LH, Mamelak AJ. Review of actinic keratosis. Part I: Etiology, epidemiology and clinical presentation. J Drugs Dermatol 2010; 9: 1125-32.

2. Werner RN, Stockfleth E, Connolly SM, et al. Evidence- and consensus-based (S3) Guidelines for the treatment of actinic keratosis - International League of Dermatological Societies in cooperation with the European Dermatology Forum - Short version. J Eur Acad Dermatol Venereol 2015; 29: 2069-79.

3. Ackerman AB, Mones JM. Solar (actinic) keratosis is squamous cell carcinoma. Br J Dermatol 2006; 155: 9-22.

4. Röwert-Huber J, Patel MJ, Forschner T, et al. Actinic keratosis is an early in situ squamous cell carcinoma: a proposal for reclassification. Br J Dermatol 2007; 156: 8-12.

5. Green A, Beardmore G, Hart V, et al. Skin cancer in a Queensland population. J Am Acad Dermatol 1988; 19: 1045-52.

6. Uehara F, Miwa S, Tome Y, et al. Imaging of UVB and UVCinduced DNA damage repair in cancer cells in Gelfoam his toculture and minimal cancer in mice. Cancer Res 2014; 74: 2403.

7. Grant WB. Ecological studies of the UVB-vitamin D-cancer hypothesis. Anticancer Res 2012; 32: 223-36.

8. Roewert-Huber J, Stockfleth E, Kerl H. Pathology and pathobiology of actinic (solar) keratosis - an update. Br J Dermatol 2007; 157: 18-20.

9. Włodarkiewicz A, Narbutt J, Adamski Z, et al. Actinic keratosis - state of art. Statement of experts of Polish Dermatological Society. Dermatol Rev/Przegl Dermatol 2014; 101: 156-67.

10. Marks R, Foley P, Goodman G, et al. Spontaneous remission of solar keratoses: the case for conservative management. Br J Dermatol 1986; 115: 649-55.

11. Cockerell CJ. Histopathology of incipient intraepidermal squamous cell carcinoma ("actinic keratosis"). J Am Acad Dermatol 2000; 42: 11-7.

12. Williams CS, Mann M, DuBois RN. The role of cyclooxygenases in inflammation, cancer, and development. Oncogene 1999; 18: 7908-16.
13. Hafner C, Reichle A, Vogt T. New indications for established drugs: combined tumor-stroma-targeted cancer therapy with PPARgamma agonists, COX-2 inhibitors, mTOR antagonists and metronomic chemotherapy. Curr Cancer Drug Targets 2005; 5: 393-419.

14. Reddy BS, Nayini J, Tokumo K, et al. Chemoprevention of colon carcinogenesis by concurrent administration of piroxicam, a nonsteroidal antiinflammatory drug with $D, L-$ alpha-difluoromethylornithine, an ornithine decarboxylase inhibitor, in diet. Cancer Res 1990; 50: 2562-8.

15. Algra AM, Rothwell PM. Effects of regular aspirin on longterm cancer incidence and metastasis: a systematic comparison of evidence from observational studies versus randomised trials. Lancet Oncol 2012; 13: 518-27.

16. Fürstenberger G, Gross M, Mark SF. Eicosanoids and multistage carcinogenesis in NMRI mouse skin: role of prostaglandins $\mathrm{E}$ and $\mathrm{F}$ in conversion (first stage of tumor promotion) and promotion (second stage of tumor promotion). Carcinogenesis 1989; 10: 91-6.

17. Anuja K, Roy S, Ghosh C, et al. Prolonged inflammatory microenvironment is crucial for pro-neoplastic growth and genome instability: a detailed review. Inflamm Res 2016; 66: 119-28.

18. Nissinen L, Farshchian M, Riihilä P, Kähäri VM. New perspectives on role of tumor microenvironment in progression of cutaneous squamous cell carcinoma. Cell Tissue Res 2016; 365: 691-702.

19. Kagoura M, Toyoda M, Matsui C, Morohashi M. Immunohistochemical expression of cyclooxygenase- 2 in skin cancers. J Cutan Pathol 2001; 28: 298-302.

20. Buckman S. COX-2 expression is induced by UVB exposure in human skin: implications for the development of skin cancer. Carcinogenesis 1998; 19: 723-9.

21. Kuźbicki Ł, Lange D, Stanek-Widera A, Chwirot BW. Different expression of cyclooxygenase-2 (COX-2) in selected nonmelanocytic human cutaneous lesions. Folia Histochem Cytobiol 2011; 49: 381-8.

22. Amirnia M, Babaie-Ghazani A, Fakhrjou A, et al. Immunohistochemical study of cyclooxygenase-2 in skin tumors. J Dermatol Treat 2014; 25: 380-7. 